assumed inertia of developmental systems. applicable to all examples of stasis irrespective of species or conditions. This says that no evolutionarily significant shift in the average phenotype of a population took place because the ecological and developmental conditions were such that mutationally deviant individuals were continually selected against. This explanation, whilst theoretically self-evident. presupposes a uniformity of environment over vast distances of time and space that surely must be unacceptable to ecologists familiar with the ever-changing web of relationships that go into the making of ecological niches. It is difficult to accept that the heterogeneity of niches. once set up. is so stable and no longer subject to further subdivision that the usual ecological pressures, forcing variant individuals to selectively adapt to progressively specialized niches, no longer exist. Furthermore, developmental inertia is becoming the panacea for all our unsolved problems. When we don't want any appreciative directional selection to be taking place we appeal to the severity of genetic and epigenetic constraints implicated in the development of a single-celled zygote to a multicellular adult. When we do need to explain, nevertheless, that evolution is seen to be taking place sometimes, such constraints miraculously disappear.

There are other potential explanations, Darwinian and non-Darwinian, for stasis and its occasional interruption, which have not been fully explored, as yet ${ }^{3.4}$. However, notwithstanding all such attempts, our general ignorance of developmental genetics and our even greater ignorance of the ecological flux and microheterogeneity of past environments, simply make it premature to wage polemical wars and to demote contemporary observations and their interpretation as "a technical parochial affair if ever there was one". The writings of Darwin, and indeed those that contributed to the formulation of the neo-Darwinian synthesis in the 1930s, are not Old Testament tracts to be poured over by armchair exegesists. They cannot supply, without resorting to too many ad hoc assumptions, all the answers to the multiple causes of the ebb and flow of evolution. This is relevant not only to the tempo of change observed in the geological record, but also to the evolutionary consequences of the unexpected molecular dynamics of the genes themselves. Physics moved on from Newton, and biology might need to move on from Darwin, if we are to explain, satisfactorily, all that we observe.

Dawkins is defending a tradition which hardly needs defending (for contemporary theories whilst non-Darwinian are not anti-Darwinian, despite the journalistic, and occasional professional, recourse to hype), when at the same time he is getting a little of his own back on the justifiable attacks on his own interesting little misjudgement of the "gene as the unit of selection" (selfish gene). Ironically, this technical blunder. (despite Dawkins' protestation that it "has enriched the synthetic theory"). stood Darwin on his head and sent many a true Darwinian rushing to set the old gent firmly on his feet. Dawkins' style seems to be a self-conscious attempt to emulate the rapier-like thrusts of Sir Peter Medawar in his prime, who demolished bogus scientists with unremitting glee. Unlike Sir Peter, however, we are left with the image of a man who in largely ignoring the real scientific issue under review, is shadow boxing with himself: a stance not much help for an informed evaluation of evolutionary processes.

Department of Genetics,

University of Cambridge,

Cambridge $\mathrm{CB} 23 \mathrm{EH}$, UK

1. Dawkins. R. Nature 316. 68.3 (1985).

2. Charlesworth. B.. Lande. R.\& Slathin. M. Evolution 36. $474(1982)$.

Mivnard Smith. J. A. Rev: Genet. 17, 11 (1983)

4. Dover. G.A. Nature 299. III (1982).

\section{The length of myosin subfragment-one}

SIR-A recent News and Views comment on electron microscopy of crystals of isolated myosin heads (S1) emphasized that these results yielded a length supporting earlier rotary shadowed views of intact myosin and $\mathrm{S} 1$ molecules. Results obtained from X-ray scattering and (by implication) certain electron microscopic acto-S1 reconstructions which yielded shorter structures were rejected as incorrect. We contend that because of a confusion arising from comparing two different kinds of lengths, there is little or no evidence for either support or rejection.

Winkelmann et $_{\text {al }}{ }^{2}$ report at least $160 \AA$ for the 'length' of S1. Measurements from their projected images (corrected for depth) indicate that this is a contour length and that the maximum chord $\left(l_{\max }\right)$ observable within their structure is $120-140 \AA$ long. The lower limit arises from the contour drawn in Fig. $11 a$ of ref. 2. The upper limit derives from the assumption that there are crystal contacts along the long direction of the molecule. While we agree that the contour in Fig. $11 a$ probably underestimates the volume, the data do not necessarily lead to the conclusion that the missing mass is along the long direction of $\mathrm{S} 1$; the molecule could just as well pack in the crystal without contacts along this direction.

$\mathrm{X}$-ray scattering results give an $l_{\max }$ $=120 \pm 10 \AA$ and acto-S1 reconstructions give $l_{\max }=115-150 \AA$ (see Table 1 of ref. 4) which are consistent with the crystal values. (The largest value in this range arises from scallop S1 with regulatory light chain.) Although these results, as well as the crystal results, could underestimate the true length of the myosin head (for different reasons), the current $\mathrm{S} 1$ crystal results do not require that the $l_{\max }(190 \AA)$ of the entire myosin head ${ }^{56}$ or $\mathrm{S}^{7}$ esti- mated from rotary shadowing projections be correct. It is nevertheless encouraging that there are features of S1 on which nearly all results concur: a curved shape with most of the mass located near one end.

ROBERTMENIDELSON Department of Biochemistry

and Biophysics,

University of California.

San Francisco, California 94143. USA

1. Craig, R. Nunure 316. 16 (1985.5)

Winkelmann. D.A.. Mekecl. H. \& Riwnent.I. J. molec Biml. 487 (1985)

Mindelson R A Krecoschmar K. Biochemistr: 19. $11.30(198(1)$.

Mendelson, R.A. \& Wagner. P.D. J. motec Biol. 181, f87 Mende

Elliott. G. \& Offer. G. J. molec. Biol. 123, $5(15$ (1977).

6. Knight. P. \& Trinick, J. J. molsc: Biol. 177. 261 (1984).

Flicker. P.F.. Willimann. T. \& Vibert. P. J. molec. Biol 169. 723 (1985).

\section{Control of the cell cycle in yeast}

SIR-In a recent News and Views article on yeast cell biology (Nature 316, 678), I. Herskowitz states that mutations in yeast that result in $G_{1}$ arrest with reduced protein synthesis are relatively unlikely to reflect specific cell-cycle controls, in contrast with mutations such as $c d c 28$, which induce $G_{1}$ arrest without reduced protein synthesis. There are several reasons for viewing this statement with caution.

First, successfully meeting the criteria of continued protein synthesis for $G_{1}$ arrest mutants does not exclude the possibility that the mutation may effect some continuous aspect of metabolism unrelated to growth control per se. For example, a mutation in the gene encoding ribonucleotide reductase could in principle mimic the effect of exogenously applied hydroxyurea, which can lead to $G_{1}$ arrest with continued protein synthesis.

Second, there is a wide body of evidence which suggests that attainment of a critical amount of protein is necessary for traversal of the $G_{1}$ period, and it seems relatively safe to assume that cell cycle control points operate both before and after such an event. There is no $a$ priori reason that physiologically important cell cycle controls are more likely to operate after this event; in mammalian cells, $G_{1}$ arrest induced by depletion of polypeptide growth factors is usually accompanied by a marked fall in protein synthesis.

Finally, two of the mutations in $S$. cerevisiae that lead to $G_{1}$ arrest with reduction of protein synthesis, $c d c 25$ and $c d c 35$, are now thought to be specific signal transduction components of the cAMP pathway. These gene products might exert their growth-regulatory effect by influencing protein synthesis, or a feedback mechanism might slow protein synthesis in response to inactivation of these gene products. In any case, any comprehensive view of cell cycle control should not exclude such regulatory proteins from consideration. SCOTT POWERS

Cold Spring Harbor Laboratory, New York 11724, USA 